\title{
Enhancement of solar cell performance by employing planar spectral converters
}

Cite as: Appl. Phys. Lett. 87, 151117 (2005); https://doi.org/10.1063/1.2099532

Submitted: 27 June 2005. Accepted: 07 September 2005. Published Online: 07 October 2005

W. G. J. H. M. van Sark

\section{ARTICLES YOU MAY BE INTERESTED IN}

Improving solar cell efficiencies by down-conversion of high-energy photons Journal of Applied Physics 92, 1668 (2002); https://doi.org/10.1063/1.1492021

Detailed Balance Limit of Efficiency of p-n Junction Solar Cells

Journal of Applied Physics 32, 510 (1961); https://doi.org/10.1063/1.1736034

Application of $\mathrm{NaYF}_{4}: \mathrm{Er}^{3+}$ up-converting phosphors for enhanced near-infrared silicon solar cell response

Applied Physics Letters 86, 013505 (2005); https://doi.org/10.1063/1.1844592

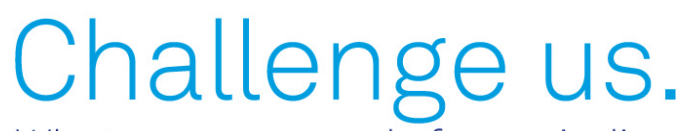

What are your needs for periodic signal detection?

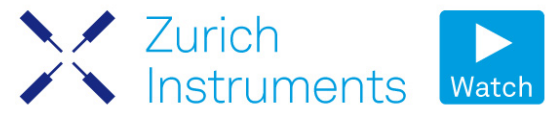

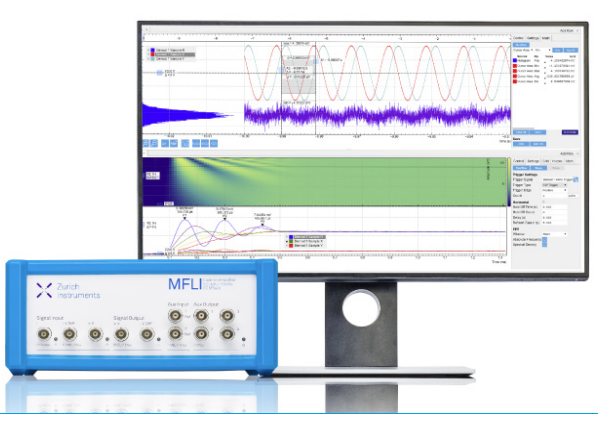

87, 151117 


\title{
Enhancement of solar cell performance by employing planar spectral converters
}

\author{
W. G. J. H. M. van Sark ${ }^{a)}$ \\ Department of Science, Technology and Society, Copernicus Institute for Sustainable Development \\ and Innovation, Heidelberglaan 2, NL-3584 CS Utrecht, the Netherlands
}

(Received 27 June 2005; accepted 7 September 2005; published online 7 October 2005)

\begin{abstract}
The effect on solar cell performance of planar converters containing quantum dots (QDs) as wavelength-shifting entities on top of multicrystalline silicon cells was investigated by means of model studies with varying incident spectra. These included global, direct, and diffuse spectra with Air Mass (AM) values ranging from 1 to 10. In case of AM1.5, a planar converter with QDs emitting at $603 \mathrm{~nm}$ yields a short-circuit current increase of $6.3 \%, 9.6 \%$, and $28.6 \%$ for direct, global, and diffuse irradiation, respectively, as a result of the larger blue/green content of diffuse spectra with respect to direct and global ones. For other AM values, similar results are calculated, with a lower increase toward high AM values. (C) 2005 American Institute of Physics. [DOI: 10.1063/1.2099532]
\end{abstract}

One of the major challenges photovoltaic research is facing today is to increase solar cell conversion efficiency by making better use of the solar spectrum. ${ }^{1,2}$ Besides the development of multijunction cells, deployment of spectral conversion, either down- or upward, ${ }^{3}$ has been pursued. Embedding both down- and upconversion materials together in one matrix would, in its optimized form, lead to conversion of the solar spectrum to a near-monochromatic one. Solar cell design would then need to be optimized for the resultant near-monochromatic spectrum, and solar cell efficiencies are expected to be far beyond the present $5-15 \%$ values of commercially available cells. As a first step in this direction, research has been performed on the so-called luminescent concentrator (LC). ${ }^{4}$ It was reported that the availability of semiconductor quantum dots (QDs) as downconverting materials in LCs would make higher conversion efficiencies possible. $^{5}$

Instead of using LCs, planar converters containing wavelength-shifting entities on top of standard solar cells may be a simple and cheap application of spectral downconversion for the enhancement of solar cell performance. The wavelength-shifting entities should shift the wavelengths where the spectral response is low to wavelengths where the spectral response is high. QDs present in a plastic layer on top of a solar cell modify the incident Air Mass (AM) 1.5 global (AM1.5G) spectrum such that the short-circuit current of the underlying multicrystalline silicon (mc-Si) increases by about $10 \% .^{6}$ As diffuse spectra are blueshifted with respect to direct and global ones, it is expected that the use of a downconverter yields better solar cell performance compared to the standard AM1.5G incident spectrum. This is of importance for areas at moderate-to-large latitudes as the amount of diffuse irradiation may equal that of direct irradiation on an annual basis, ${ }^{7}$ while direct irradiation conditions prevail in areas broadly located around the equator. In this letter, we will investigate the effect of various incident spectra on performance of solar cells on to which planar spectral converters are applied. This entails global, direct, and diffuse spectra of AM values ranging from 1 to 10 .

\footnotetext{
${ }^{a)}$ Electronic mail: w.g.j.h.m.vansark@chem.uu.nl
}

For solar cell performance testing, the AM1.5G standard is widely used, see, e.g., Ref. 8. This global spectrum consists of a direct and a diffuse part, with contributions of $82 \%$ and $18 \%$, respectively. In this letter, spectra were calculated for clear sky conditions using the solar irradiance model SPECTRAL2 (Ref. 9) with standard atmospheric values: Turbidity 0.27 at $0.5 \mu \mathrm{m}$; ground albedo 0.2 ; precipitable water $1.42 \mathrm{~cm}$; total ozone $0.34 \mathrm{~atm} \mathrm{~cm}$. For ease of calculation, longitude and latitude were both set at zero degrees, hence AM1 spectral conditions are present at noon at the vernal equinox (March 23). The time of day was varied from noon toward the morning to calculate global, direct, and diffuse spectra at AM values between 1 and 10. The irradiation intensity is found to decrease from $1090 \mathrm{~W} / \mathrm{m}^{2}$ at AM1 to $176 \mathrm{~W} / \mathrm{m}^{2}$ at AM10. In addition, the diffuse fraction in the wavelength range of $300-593 \mathrm{~nm}$ increases from $30 \%$ at AM1 to $70 \%$ at AM10. Interestingly, the peak positions of the diffuse spectra remain about constant at $450 \mathrm{~nm}$, while those of the direct and global spectra redshift from 550 and 480 , respectively, to $860 \mathrm{~nm}$.

The configuration studied is a highly transparent layer containing CdSe QDs on top of a mc-Si solar cell. ${ }^{10}$ The size of the QDs is taken such that both blue and green light is absorbed and emitted in the red, which corresponds to an optimum spectral response of the solar cell. Depending on the QD concentration also unabsorbed blue and green light enters the solar cell. For solar cell simulation, we take the standard baseline $n-p-p^{+}$mc-Si cell developed at the Energy Research Centre of the Netherlands, ${ }^{10}$ which has parameters that are typical of low-cost commercial products. It has an area of $10 \times 10 \mathrm{~cm}^{2}$, a thickness of $300 \mu \mathrm{m}$, and a shallow diffused emitter of $50 \Omega / \mathrm{sq}$. The front broadband reflectance is $9 \%$ across the solar spectrum, as a result of the front surface antireflection coating $(71 \mathrm{~nm}$ thick silicon nitride with a refractive index $n=2.1$ ). The thickness of the back-surface field is $9 \mu \mathrm{m}$; the $p^{+}$-doping level is of $4 \times 10^{18} \mathrm{~cm}^{-3}$. The performance of the solar cell is simulated with the simulation programme PC1D (version 5.8). ${ }^{11}$ The calculated performance parameters (short-circuit current $I_{\mathrm{sc}}$, open-circuit voltage $V_{\mathrm{oc}}$, fill factor $F F$, and efficiency $\eta$ ) are $31.2 \mathrm{~mA} / \mathrm{cm}^{2}, 0.603 \mathrm{~V}$, 0.771 , and $14.5 \%$, respectively, for $\mathrm{AM} 1.5 \mathrm{G}$ standard irradiation. 


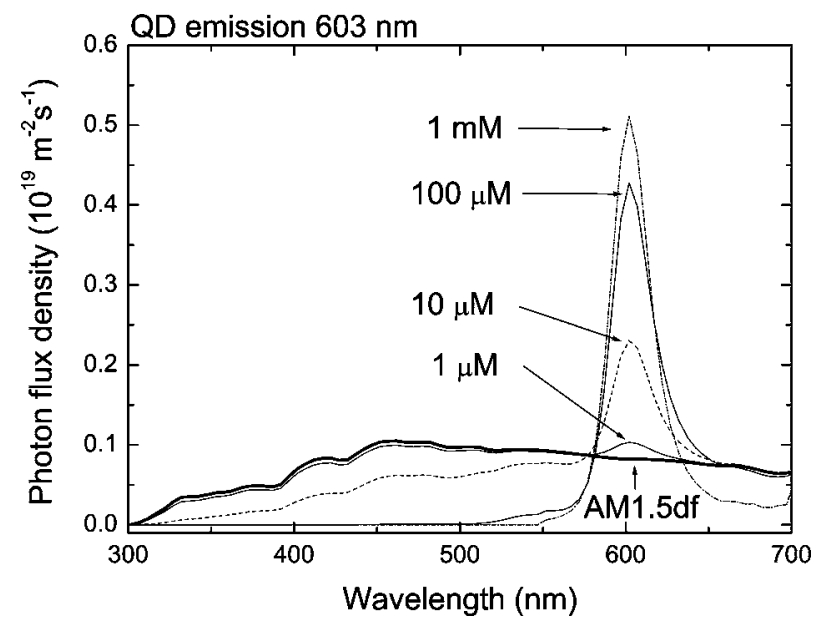

FIG. 1. Calculated modified AM1.5 diffuse spectra for QD concentrations from $1 \mu \mathrm{M}$ to $1 \mathrm{nM}$.

Upon absorption of photons by QDs, the incident spectrum, $\Phi_{s}(\lambda)$, is modified. The amount of absorbed photons $\Phi_{a}(\lambda)$ is determined from the QD absorption spectrum, which depends on QD size, their concentration in the converter layer, and thickness of this layer. This absorbed amount is subtracted from the incident spectrum: $\Phi_{s a}(\lambda)=\Phi_{s}(\lambda)-\Phi_{a}(\lambda)$. The QDs re-emit light at a redshifted wavelength, and the amount of emitted photons $\Phi_{e}(\lambda)$ is calculated from the QD emission spectrum. A quantum efficiency of 0.8 is assumed, ${ }^{12,13}$ as well as the assumption that three-quarters of the emitted photons are directed toward the underlying solar cell, due to internal reflection in the converter layer. ${ }^{14}$ Note that these data reflect an ideal case. The amount of emitted photons is then added to the modified AM1.5G spectrum, $\Phi_{s a e}(\lambda)=\Phi_{s a}(\lambda)+\Phi_{e}(\lambda)$, and the resulting spectrum serve as input for the solar cell simulation model.

Absorption of photons is calculated by using the Lambert-Beer equation: The photon flux density $\Phi(x, \lambda)$ after passing a distance $x$ in a film with absorption coefficient $\alpha(\lambda)$ equals $\Phi(x, \lambda)=\Phi^{0}(\lambda) \exp [-\alpha(\lambda) x]$, with $\Phi^{0}(\lambda)$ as the incident photon flux density. The exponential term $\alpha(\lambda) x$ equals $\varepsilon_{\lambda} C D$, with $\varepsilon_{\lambda}$ as the molar extinction coefficient $\left(\mathrm{M}^{-1} \mathrm{~cm}^{-1}\right), C$ as the chromophore concentration $(\mathrm{M})$, and $D$ as the thickness of the film $(\mathrm{cm})$. The molar extinction coefficient is determined by scaling the normalized absorption spectrum such that the molar extinction coefficient $\varepsilon_{\lambda}$ at $350 \mathrm{~nm}$ equals $\varepsilon_{\lambda}=1.438 \times 10^{26} a^{3}$ as reported by Leatherdale $e t a l .{ }^{15}$ The QD radius $a$ is determined from the absorption maximum and the known relation with particle diameter. ${ }^{16}$ The QD absorption maximum was $594 \mathrm{~nm}$, corresponding to a QD radius of $2.1 \mathrm{~nm}$. The QD concentration is varied from $1 \mathrm{nM}$ to $10 \mathrm{mM}$ with a converter thickness $D$ of $0.1 \mathrm{~cm}$. As the product $C D$ determines the amount of absorption and spectral change, optimum values for QD concentration are related to the thickness of the converter.

The effect of spectral conversion by the inclusion of various concentrations of QDs on the AM1.5 diffuse incident spectrum is shown in Fig. 1. Starting at a concentration of $1 \mu \mathrm{M}$, an appreciable amount of photons is absorbed in the blue part of the AM1.5 diffuse spectrum, while the modified spectrum is increased at the QD emission wavelength $(603 \mathrm{~nm})$. For higher concentrations, this effect is clearly

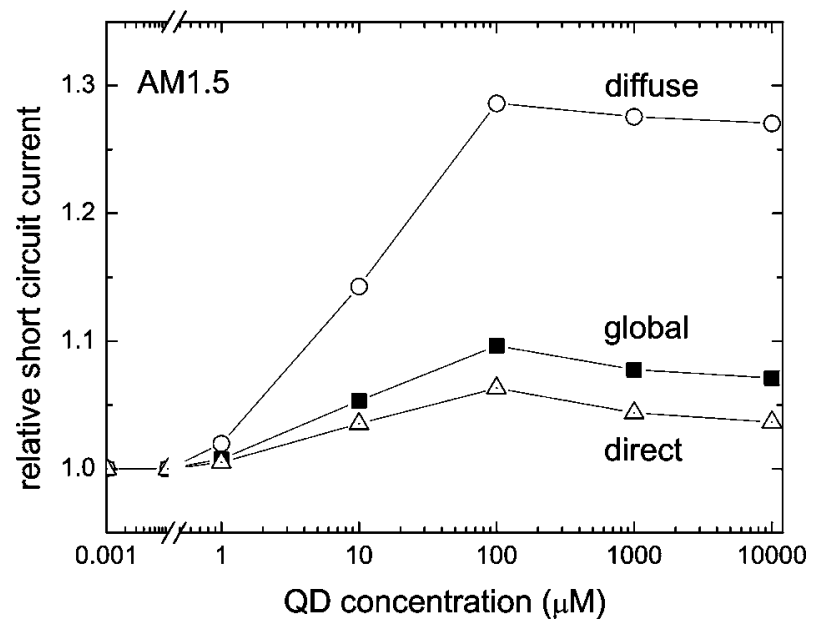

FIG. 2. Relative short-circuit current as a function of QD concentration for the AM1.5 global direct, and diffuse spectra.

much stronger. The effect of QD inclusion on all spectra is similar to the one shown in Fig. 1.

Figure 2 shows the relative change in short-circuit current as a function of QD concentration. Between $1 \mathrm{nM}$ and $1 \mu \mathrm{M}$, no appreciable effects are observed. A maximum relative increase is obtained for all spectra at a concentration of $100 \mu \mathrm{M}$. This increase is $9.6 \%, 6.3 \%$, and $28.6 \%$ for the global, direct, and diffuse spectrum, respectively. The increase in case of the global spectrum (9.6\%) compares well with the linear combination of data obtained for diffuse and direct spectra $(10.2 \%)$, taking into account their fractions in the global spectrum. For higher concentrations, the effect levels off. Here, the beneficial effect is counteracted by the increased absorption due to the high concentration. It should be noted that in practice the results for the highest concentrations are less reliable due to the fact that re-absorption is not taken into account in our simulations. The calculated relative increase in short-circuit current is also found for maximum generated power $P_{\max }$. The open-circuit voltage and fill factor only slightly decrease over the full range from $1 \mathrm{~nm}$ to $10 \mathrm{mM}$ (not shown).

For the whole range of global, direct, and diffuse AM1-10 spectra, we simulated solar cell performance changes for the QDs emitting at $603 \mathrm{~nm}$ for a concentration range from $1 \mathrm{~nm}$ to $10 \mathrm{mM}$. The results show similar behavior as a function of QD concentration as in the case of AM1.5 (Fig. 2), i.e., the relative change in short-circuit current is found maximal for a concentration of $100 \mu \mathrm{M}$. For this optimal concentration, results are shown in Fig. 3, where the maximum relative current increase is depicted as a function of AM for global, direct, and diffuse spectra. The increase in short-circuit current is largest for the AM1 diffuse spectrum, i.e., $31.4 \%$, which decreases to $20.2 \%$ for AM10. For global spectra these values are $11.9 \%$ and $1.5 \%$ for AM1 and AM10, respectively. In the case of direct spectra, the increase in short-circuit current changes into a decrease between AM5 and AM6. The maximum increase is $8.2 \%$ for AM1; for AM10 the decrease is $-1.1 \%$.

The results can be explained by a combination of factors. First, the peak positions of direct and global spectra redshift, while the diffuse spectra do not shift and remain constant at $450 \mathrm{~nm}$. Second, the diffuse fraction in the wavelength region of interest for the QDs used here (i.e., photons with wavelengths in the range of $300-593 \mathrm{~nm}$ can be absorbed) 


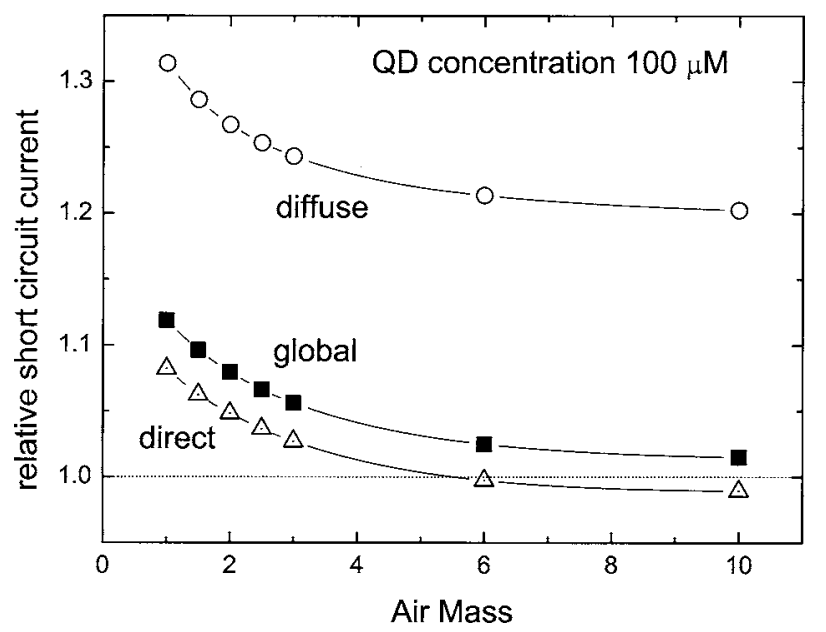

FIG. 3. Maximum relative short-circuit as a function of AM for global, direct, and diffuse spectra at a QD concentration of $100 \mu \mathrm{M}$.

increases from $30 \%$ at AM1 to $70 \%$ at AM10. Third, as shown in Fig. 4, the spectral content for diffuse spectra in this wavelength region relative to the total content is much larger than that of global and direct spectra. As a consequence, beneficial effects on performance for diffuse spectra are much more pronounced that for global and direct spectra.

In summary, the inclusion of a planar converter containing wavelength-shifting entities, such as QDs, allows for a better use of the solar spectrum. QDs shift the wavelengths where the spectral response of a solar cell is low to wavelengths where the spectral response is high. In this letter, we showed that QDs-with a center emission wavelength of $603 \mathrm{~nm}$ included in a planar converter on top of a mc-Si solar cell-are capable of increasing short-circuit current by

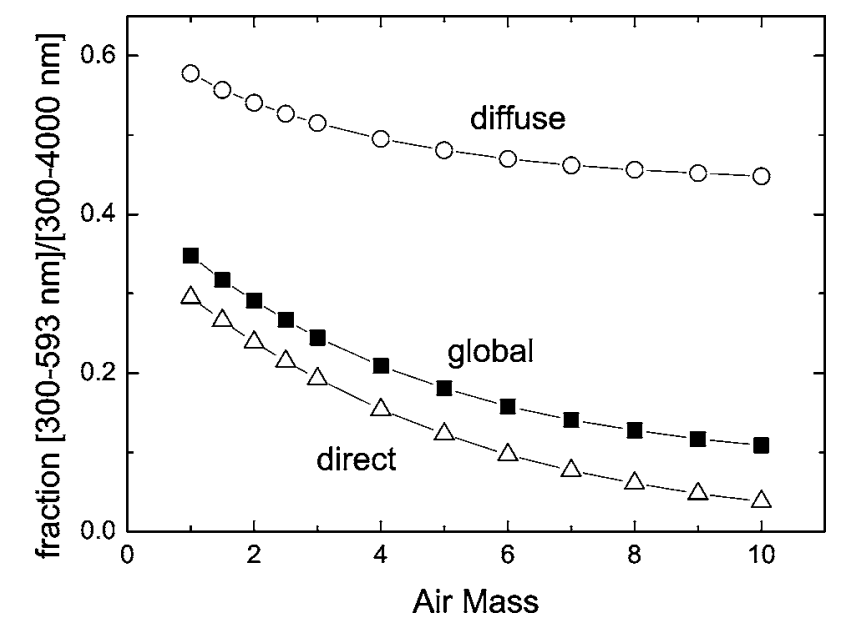

FIG. 4. Spectra content in the wavelength region $300-593 \mathrm{~nm}$ relative to the total content as a function of AM for global, direct, and diffuse spectra.
$6.3 \%, 9.6 \%$ and $28.6 \%$ for AM1.5 direct, global, and diffuse spectra, respectively. For other AM values, similar results are calculated, with a lower increase toward high AM values. The large increase in short-circuit current for diffuse spectra is a result of a larger blue/green content of diffuse spectra compared to direct and global spectra in combination with the blue response of solar cells that generally is worse than their red response. The implications for the use of planar converters in outdoor conditions are clear, especially in regions on earth where diffuse spectral conditions are as important as direct ones. With planar and/or LCs, more use can be made of diffuse spectra, leading to an overall improved performance.

The author gratefully acknowledges Dr. D. R. Myers (National Renewable Energy Laboratory, Golden CO, USA) for making available the SPCTRL2 model in spreadsheet format, and the European Commission for financial support as part of the Framework 6 integrated project FULLSPECTRUM (Contract No. SES6-CT-2003-502620).

${ }^{1}$ M. A. Green, Third Generation Photovoltaics, Advanced Solar Energy Conversion (Springer, Berlin, Germany, 2003).

${ }^{2}$ A. Luque, A. Martí, A. Bett, V. M. Andreev, C. Jaussaud, J. A. M. van Roosmalen, J. Alonso, A. Räuber, G. Strobl, W. Stolz, C. Algora, B. Bitnar, A. Gombert, C. Stanley, P. Wahnon, J. C. Conesa, W. G. J. H. M. van Sark, A. Meijerink, G. P. M. van Klink, K. Barnham, R. Danz, T. Meyer, I. Luque-Heredia, R. Kenny, C. Christofides, G. Sala, and P. Benítez, Sol. Energy Mater. Sol. Cells 87, 467 (2005).

${ }^{3}$ T. Trupke, M. A. Green, and P. Würfel, J. Appl. Phys. 92, 1668 (2002); T. Trupke, M. A. Green, and P. Würfel, ibid. 92, 4117 (2002).

${ }^{4}$ A. Goetzberger and W. Greubel, Appl. Phys. 14, 123 (1977).

${ }^{5}$ A. J. Chatten, K. W. J. Barnham, B. F. Buxton, N. J. Ekins-Daukes, and M. A. Malik, Sol. Energy Mater. Sol. Cells 75, 363 (2003).

${ }^{6}$ W. G. J. H. M. van Sark, A. Meijerink, R. E. I. Schropp, J. A. M. van Roosmalen, and E. H. Lysen, Sol. Energy Mater. Sol. Cells 87, 395 (2005).

${ }^{7}$ M. Iqbal, An Introduction to Solar Radiation (Academic, New York, 1983).

${ }^{8}$ D. R. Myers, K. Emery, and C. Gueymard, J. Sol. Energy Eng. 126, 567 (2004).

${ }^{9}$ R. E. Bird and C. Riordan, J. Clim. Appl. Meteorol. 25, 87 (1986).

${ }^{10} \mathrm{C}$. Tool (personal communication).

${ }^{11}$ P. A. Basore and D. A. Clugston, Proceedings of the 25th IEEE Photovoltaic Specialists Conference, Washington, D.C. (IEEE, New York, 1996), p. 377.

${ }^{12}$ C. De Mello Donegá, S. G. Hickey, S. F. Wuister, D. Vanmaekelbergh, and A. Meijerink, J. Phys. Chem. B 107, 489 (2003).

${ }^{13}$ L. Qu and X. Peng, J. Am. Chem. Soc. 124, 2049 (2002).

${ }^{14}$ A. J. Chatten, K. W. J. Barnham, B. F. Buxton, N. J. Ekins-Daukes, and M. A. Malik, Proceedings of the Third World Congress on Photovoltaic Energy Conversion WPEC-3, Osaka, Japan (WCPE-3, Osaka, Japan, 2003), p. 2567.

${ }^{15}$ C. A. Leatherdale, W.-K. Woo, F. V. Mikulec, and M. G. Bawendi, J. Phys. Chem. B 106, 7619 (2002).

${ }^{16}$ B. O. Dabbousi, J. Rodriguez-Viejo, F. V. Mikulec, J. R. Heine, H. Mattoussi, R. Ober, K. F. Jensen, and M. G. Bawendi, J. Phys. Chem. B 101, 9463 (1997). 